\title{
Analysis of Conduction Band Offset Variation on the Electrostatics of UTB Devices through the Modified Effective Mass Approximation (mEMA)
}

This paper was downloaded from TechRxiv (https://www.techrxiv.org).

\section{LICENSE}

CC BY-NC-SA 4.0

SUBMISSION DATE / POSTED DATE

$12-01-2022$ / 18-01-2022

\section{CITATION}

Kansal, Harshit; Vilochan Mishra, Nalin; Solanki, Ravi; Medury, Aditya S (2022): Analysis of Conduction Band Offset Variation on the Electrostatics of UTB Devices through the Modified Effective Mass Approximation (mEMA). TechRxiv. Preprint. https://doi.org/10.36227/techrxiv.18262490.v1

$\mathrm{DOI}$ 


\section{SUPPLEMENTARY MATERIAL}

\section{A. Supplement S1:}

Tables showing effective mass parameters for various gate voltages with energy correction terms corresponding to ground and other excited states ensuring excellent agreement with TB method for channel thickness varying from $2 \mathrm{~nm}$ to $10 \mathrm{~nm}$ and for oxide thickness $1 \mathrm{~nm}$ and $5 \mathrm{~nm}$. (Considering no tunneling)

TABLE I: $T_{s i}=2 n m$

\begin{tabular}{cccccc|ccccc}
\multicolumn{3}{c}{$T_{\text {ox }}=1 \mathrm{~nm}$} \\
\hline \hline Voltage $(\mathbf{V})$ & $\mathbf{m}_{\mathbf{d o s}}$ & $\mathbf{m}_{\text {st }}$ & $\mathbf{m}_{\mathbf{0 x}}$ & $\Delta E_{0}$ & $\Delta E_{1}$ & $\mathbf{m}_{\text {dos }}$ & $\mathbf{m}_{\text {st }}$ & $\mathbf{m}_{\mathbf{0 x}}$ & $\Delta E_{0}$ & $\Delta E_{1}$ \\
\hline 0.087 & 0.23 & 0.76 & 0.9 & 0.173 & -0.07 & 0.23 & 0.74 & 0.4 & 0.173 \\
0.287 & 0.23 & 0.76 & 0.9 & 0.172 & -0.07 & 0.23 & 0.74 & 0.4 & 0.173 & -0.08 \\
0.661 & 0.23 & 0.75 & 0.9 & 0.170 & -0.07 & 0.23 & 0.74 & 0.4 & 0.169 & -0.08 \\
1.040 & 0.23 & 0.75 & 0.9 & 0.170 & -0.05 & 0.23 & 0.72 & 0.4 & 0.169 & -0.08 \\
1.618 & 0.23 & 0.73 & 0.9 & 0.169 & -0.02 & 0.23 & 0.70 & 0.4 & 0.169 & -0.08 \\
2.001 & 0.23 & 0.72 & 0.9 & 0.169 & -0.02 & 0.23 & 0.70 & 0.4 & 0.169 & -0.08 \\
\hline \hline
\end{tabular}

TABLE II: $T_{s i}=3 n m$

\begin{tabular}{|c|c|c|c|c|c|c|c|c|c|c|}
\hline \multicolumn{6}{|c|}{$T_{o x}=1 \mathrm{~nm}$} & \multicolumn{5}{|c|}{$T_{o x}=5 \mathrm{~nm}$} \\
\hline Voltage (V) & $\boldsymbol{m}_{d o s}$ & $\mathbf{m}_{\text {st }}$ & $\mathbf{m}_{\mathbf{0 x}}$ & $\Delta E_{0}$ & $\Delta E_{1}$ & $\mathbf{m}_{\mathrm{dos}}$ & $\mathbf{m}_{\mathrm{st}}$ & $\mathbf{m}_{\mathbf{0 x}}$ & $\Delta E_{0}$ & $\Delta E_{1}$ \\
\hline 0.087 & 0.23 & 0.56 & 0.9 & 0.168 & 0.00 & 0.23 & 0.55 & 0.4 & 0.166 & 0 \\
\hline 0.278 & 0.23 & 0.56 & 0.9 & 0.168 & 0.00 & 0.23 & 0.55 & 0.4 & 0.166 & 0 \\
\hline 0.661 & 0.23 & 0.53 & 0.9 & 0.168 & 0.00 & 0.23 & 0.55 & 0.4 & 0.166 & 0 \\
\hline 1.040 & 0.23 & 0.5 & 0.9 & 0.168 & 0.01 & 0.23 & 0.54 & 0.4 & 0.167 & 0 \\
\hline 1.618 & 0.23 & 0.47 & 0.9 & 0.168 & 0.03 & 0.23 & 0.52 & 0.4 & 0.167 & 0 \\
\hline 2.001 & 0.23 & 0.44 & 0.9 & 0.168 & 0.03 & 0.23 & 0.5 & 0.4 & 0.168 & 0 \\
\hline
\end{tabular}

TABLE III: $T_{s i}=4 n m$

\begin{tabular}{|c|c|c|c|c|c|c|c|c|c|c|}
\hline \multicolumn{6}{|c|}{$T_{o x}=1 \mathrm{~nm}$} & \multicolumn{5}{|c|}{$T_{o x}=5 \mathrm{~nm}$} \\
\hline Voltage (V) & $\mathbf{m}_{\text {dos }}$ & $\mathbf{m}_{\text {st }}$ & $\mathbf{m}_{\mathbf{0 x}}$ & $\Delta E_{0}$ & $\Delta E_{1}$ & $\mathbf{m}_{\text {dos }}$ & $\mathbf{m}_{\text {st }}$ & $\mathbf{m}_{\mathbf{0 x}}$ & $\Delta E_{0}$ & $\Delta E_{1}$ \\
\hline 0.087 & 0.23 & 0.45 & 0.9 & 0.165 & 0.052 & 0.23 & 0.4 & 0.4 & 0.160 & 0.05 \\
\hline 0.278 & 0.23 & 0.45 & 0.9 & 0.165 & 0.058 & 0.23 & 0.4 & 0.4 & 0.160 & 0.05 \\
\hline 0.661 & 0.23 & 0.43 & 0.9 & 0.166 & 0.06 & 0.23 & 0.4 & 0.4 & 0.167 & 0.05 \\
\hline 1.040 & 0.23 & 0.40 & 0.9 & 0.167 & 0.065 & 0.23 & 0.4 & 0.4 & 0.168 & 0.06 \\
\hline 1.618 & 0.23 & 0.40 & 0.9 & 0.168 & 0.090 & 0.23 & 0.4 & 0.4 & 0.168 & 0.06 \\
\hline 2.001 & 0.23 & 0.40 & 0.9 & 0.170 & 0.100 & 0.23 & 0.4 & 0.4 & 0.168 & 0.06 \\
\hline
\end{tabular}


TABLE IV: $T_{s i}=5 \mathrm{~nm}$

$T_{o x}=1 \mathrm{~nm}$

$T_{o x}=5 n m$

\begin{tabular}{cccccc|ccccc}
\hline \hline Voltage $(\mathbf{V})$ & $\mathbf{m}_{\mathbf{d o s}}$ & $\mathbf{m}_{\mathbf{s t}}$ & $\mathbf{m}_{\mathbf{0 x}}$ & $\Delta E_{0}$ & $\Delta E_{1}$ & $\mathbf{m}_{\mathbf{d o s}}$ & $\mathbf{m}_{\mathbf{s t}}$ & $\mathbf{m}_{\mathbf{0 x}}$ & $\Delta E_{0}$ & $\Delta E_{1}$ \\
\hline 0.087 & 0.23 & 0.46 & 0.9 & 0.168 & 0.102 & 0.23 & 0.44 & 0.4 & 0.168 & 0.095 \\
0.278 & 0.23 & 0.46 & 0.9 & 0.168 & 0.102 & 0.23 & 0.44 & 0.4 & 0.168 & 0.095 \\
0.661 & 0.23 & 0.46 & 0.9 & 0.168 & 0.114 & 0.23 & 0.44 & 0.4 & 0.168 & 0.108 \\
1.040 & 0.23 & 0.46 & 0.9 & 0.168 & 0.135 & 0.23 & 0.44 & 0.4 & 0.168 & 0.112 \\
1.618 & 0.23 & 0.44 & 0.9 & 0.168 & 0.168 & 0.23 & 0.44 & 0.4 & 0.168 & 0.116 \\
2.001 & 0.23 & 0.38 & 0.9 & 0.168 & 0.168 & 0.23 & 0.44 & 0.4 & 0.168 & 0.120 \\
\hline \hline
\end{tabular}

TABLE V: $T_{s i}=7 \mathrm{~nm}$

\begin{tabular}{cccccc|ccccc}
\multicolumn{3}{c}{$T_{\text {ox }}=1 \mathrm{~nm}$} \\
\hline \hline Voltage (V) & $\mathbf{m}_{\text {dos }}$ & $\mathbf{m}_{\text {st }}$ & $\mathbf{m}_{\mathbf{0 x}}$ & $\Delta E_{0}$ & $\Delta E_{1}$ & $\mathbf{m}_{\text {dos }}$ & $\mathbf{m}_{\text {st }}$ & $\mathbf{m}_{\mathbf{0 x}}$ & $\Delta E_{0}$ & $\Delta E_{1}$ \\
\hline 0.087 & 0.23 & 0.31 & 0.9 & 0.168 & 0.128 & 0.23 & 0.31 & 0.4 & 0.168 & 0.128 \\
0.278 & 0.23 & 0.30 & 0.9 & 0.168 & 0.120 & 0.23 & 0.30 & 0.4 & 0.168 & 0.128 \\
0.661 & 0.23 & 0.28 & 0.9 & 0.168 & 0.138 & 0.23 & 0.30 & 0.4 & 0.168 & 0.128 \\
1.040 & 0.23 & 0.26 & 0.9 & 0.168 & 0.158 & 0.23 & 0.27 & 0.4 & 0.168 & 0.128 \\
1.618 & 0.23 & 0.25 & 0.9 & 0.168 & 0.168 & 0.23 & 0.26 & 0.4 & 0.168 & 0.128 \\
2.001 & 0.23 & 0.25 & 0.9 & 0.168 & 0.168 & 0.23 & 0.25 & 0.4 & 0.168 & 0.128 \\
\hline \hline
\end{tabular}

TABLE VI: $T_{s i}=10 \mathrm{~nm}$

\begin{tabular}{cccccc|ccccc}
\multicolumn{3}{c}{$T_{\text {ox }}=1 \mathrm{~nm}$} \\
\hline \hline Voltage $\mathbf{( V )}$ & $\mathbf{m}_{\text {dos }}$ & $\mathbf{m}_{\text {st }}$ & $\mathbf{m}_{\mathbf{0 x}}$ & $\Delta E_{0}$ & $\Delta E_{1}$ & $\mathbf{m}_{\text {dos }}$ & $\mathbf{m}_{\text {st }}$ & $\mathbf{m}_{\text {ox }}$ & $\Delta E_{0}$ & $\Delta E_{1}$ \\
\hline 0.087 & 0.23 & 0.23 & 0.9 & 0.165 & 0.143 & 0.23 & 0.21 & 0.4 & 0.168 & 0.139 \\
0.278 & 0.23 & 0.23 & 0.9 & 0.165 & 0.143 & 0.23 & 0.21 & 0.4 & 0.168 & 0.135 \\
0.661 & 0.23 & 0.21 & 0.9 & 0.166 & 0.142 & 0.23 & 0.19 & 0.4 & 0.168 & 0.135 \\
1.040 & 0.23 & 0.19 & 0.9 & 0.167 & 0.141 & 0.23 & 0.19 & 0.4 & 0.168 & 0.135 \\
1.618 & 0.23 & 0.18 & 0.9 & 0.168 & 0.140 & 0.23 & 0.19 & 0.4 & 0.168 & 0.135 \\
2.001 & 0.23 & 0.18 & 0.9 & 0.168 & 0.140 & 0.23 & 0.18 & 0.4 & 0.168 & 0.135 \\
\hline \hline
\end{tabular}

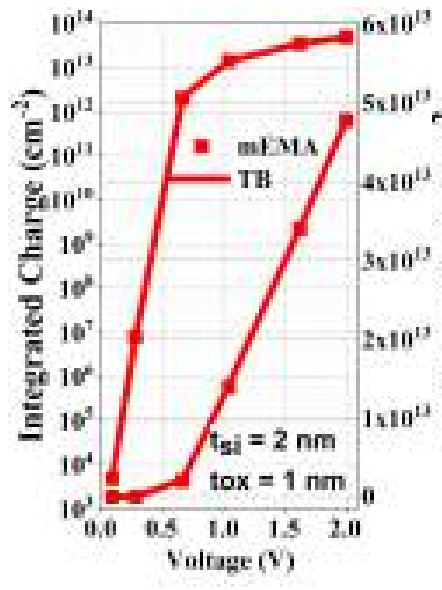

(a)

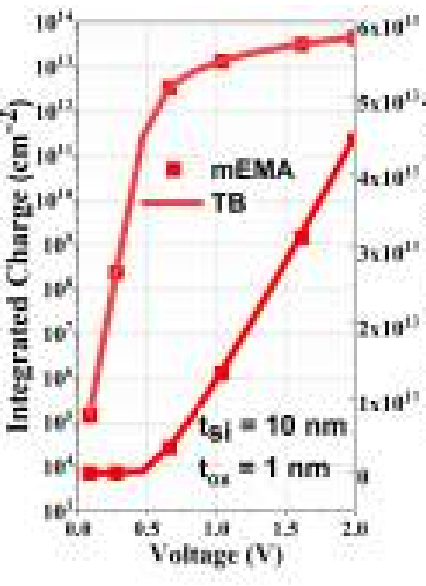

(b)

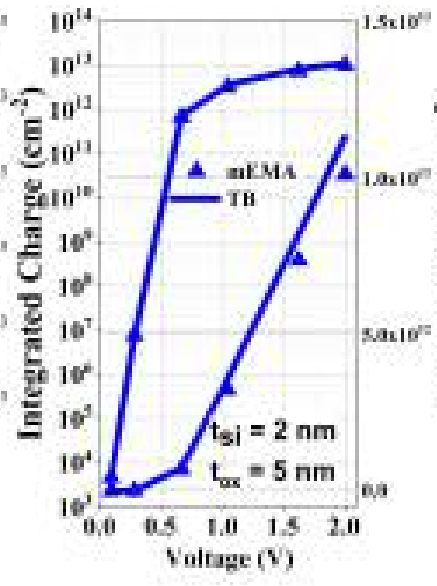

(c)

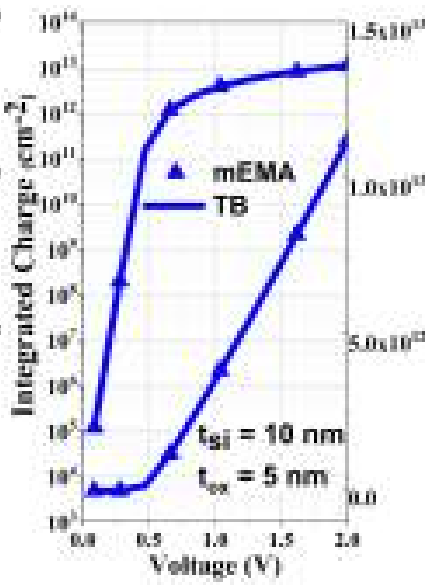

(d)

FIG. S1: Integrated Charge (Linear and Log scale) versus gate voltage with $t_{o x}=1 \mathrm{~nm}$ for (a) $t_{S i}=2 \mathrm{~nm}$ and (b) $t_{S i}=10 \mathrm{~nm}$ and $t_{o x}=5 \mathrm{~nm}$ for (a) $t_{S i}=2 \mathrm{~nm}$ and (b) $t_{S i}=10 \mathrm{~nm}$ showing good agreement between EMA and TB Method 